\title{
A Política Nacional de Educação Superior na Saúde e a Reorientação Curricular da FOUSP: Análise Comparativa do Desempenho e Percepção dos Estudantes
}

\author{
Ana Estela Haddad ${ }^{1}$, Juan Sebastian Lara Romero², Cássio José Fornazari Alencar ${ }^{3}$, Daniela \\ Prócida Raggio ${ }^{1}$, Ana Lídia Ciamponi ${ }^{1}$, Marcelo Bonecker ${ }^{1}$ \\ ${ }^{1}$ Faculdade de Odontologia da Universidade de São Paulo \\ 2Odontopediatria, University of Manchester \\ ${ }^{3}$ Odontopediatria, Universidade Paulista
}

* Autores para correspondência: aehaddad@usp.br; juansebastianlara.romero@manchester.ac.uk; cjalencar@uol.com.br; danielar@usp.br; analidia@usp.br; bonecker@usp.br

\section{RESUMO}

Este artigo apresenta um histórico recente da política nacional de educação superior e da política nacional de saúde, como contexto no qual o curso de graduação em Odontologia da Faculdade de Odontologia da USP conduziu sua reestruturação curricular. São destacados como referência o Plano Nacional de Educação (20142024), as Diretrizes Curriculares Nacionais para o curso de graduação em Odontologia, o Programa Nacional de Reorientação da Formação Profissional em Saúde (Pró-Saúde) e o Programa de Educação pelo Trabalho em Saúde (PET Saúde), como contexto a partir do qual a reestruturação foi conduzida. Relatam-se ainda a aplicação da reestruturação curricular às mudanças formuladas e implementadas na Disciplina de Odontopediatria, e o resultado da avaliação de desempenho e de percepção dos estudantes, comparando-se os currículos antes e depois da reestruturação.

Palavras-Chave: Educação Superior; Currículo; Graduação; Educação nas Profissões da Saúde; Odontopediatria..

\begin{abstract}
This paper presents a report of the national policy for higher education and health, as the context in which the undergraduate course of the Faculdade de Odontologia da USP has conducted its curricular restructuring. The reference documents for the restructuring curriculum were the National Education Plan (2014-2024), the National Curriculum Guidelines, the Pro-Saúde and PET Health Programs. It is also reported the application of curricular restructuring to the Pediatric Dentistry Discipline, as well as the result of the performance evaluation and the perception of students, comparing the curricula before and after the restructuring.
\end{abstract}

Keywords: Higher Education; Curriculum; Undergraduate Courses; Health Professionals Education; Pediatric Dentistry.

\section{Introdução}

O reconhecimento do papel estratégico das universidades para o desenvolvimento econômico e social do país e, como consequência, a necessidade de ampliar e democratizar o acesso à educação superior com qualidade têm estado na agenda da política nacional de educação na última década (BRASIL, 2014). Proporcionar esse nível de ensino a uma parcela maior da população é fator decisivo para a diminuição das desigualdades sociais e regionais, para o desenvolvimento científico e tecnológico, para a inclusão social e para a geração de trabalho e renda.

O Programa Universidade para Todos, somado ao Fies (Financiamento Estudantil) e ao Reuni (Programa Nacional de Reestruturação e Expansão das Universidades Federais no Brasil) foram responsáveis por a população universitária 
ter mais do que dobrado entre 2003 e 2010, passando de 3 milhões para 7 milhões de estudantes no ensino superior. A expansão promovida pelo Reuni correspondeu a 200 mil novas vagas de ingresso a cada ano, a abertura de concursos para a contratação de 28 mil novos docentes na rede federal em todo o país, 126 novos campi criados no interior, longe das capitais, em municípios afastados e espalhados em diferentes localidades pelo território nacional, promovendo a interiorização do desenvolvimento. Além disso, os cursos de graduação criados foram, em geral, escolhidos a partir da vocação e das atividades produtivas já existentes nas respectivas comunidades.

A expansão com qualidade está ancorada na criação do Sistema Nacional de Avaliação da Educação Superior - Sinaes (BRASIL, 2004), cujos resultados divulgados periodicamente orientam o credenciamento e recredenciamento das Instituições de Ensino Superior (IES) e a autorização, reconhecimento e renovação de reconhecimento de cursos de graduação no Brasil. Embora as universidades estaduais não estejam obrigatoriamente vinculadas ao Sistema Federal, como é o caso da rede de universidades federais e das IES privadas, tanto a Unesp como a Unicamp, e, mais recentemente, a USP, têm participado do Sinaes, permitindo assim que se possam estabelecer parâmetros de avaliação nacionais mais amplos e elevados para a educação superior como um todo. Vale ressaltar que há um entendimento firmado na referida lei e acordado entre os Conselhos Estaduais e o Conselho Nacional de Educação de que esta aproximação e integração são desejáveis.

A estratégia 12.11 da meta 12 do PNE indica que devem ser fomentados estudos e pesquisas que analisem a necessidade de articulação entre formação, currículo, pesquisa e mundo do trabalho, considerando as necessidades econômicas, sociais e culturais do país. Aplicada à área da saúde, essa estratégia nos remete a alguns aspectos ligados à Constituição Federal de 1988, que estabeleceu a saúde como direito do cidadão e dever do Estado, criando, assim, o Sistema Único de Saúde (SUS).
Numa perspectiva global da análise de sistemas de saúde de acesso universal, como é o caso do SUS, está presente a preocupação com o custo crescente que decorre do uso não racional da alta tecnologia, em contraponto à necessidade de ampliar o acesso aos serviços de saúde. Particularmente no Brasil, o planejamento das ações de saúde deve considerar ainda o acúmulo e a transição do perfil socioepidemiológico da população.

No enfrentamento desses desafios colocados para que se possa consolidar o Sistema Único de Saúde, é incontornável admitir o papel central dos trabalhadores e profissionais de saúde. Em oposição a outros setores econômicos, a saúde exige trabalho intensivo e os trabalhadores nunca serão substituídos por máquinas ou tecnologia. $\mathrm{O}$ contato pessoal é indispensável: a relação profissional/paciente incorpora a essência do cuidado em saúde. A saúde não pode ser adiada, o fator tempo é essencial, e precisa ser oferecida para cada pessoa. Por maior que seja a evolução de protocolos e do conhecimento baseado em evidência, não existe previsibilidade absoluta. Muitas doenças não dependem apenas de fatores biológicos: estresse, violência, depressão, envenenamento e contaminação, hipertensão, entre outros, têm forte correlação com o ambiente e o estilo de vida (HADDAD, 2011). A compreensão dessa complexidade precisa ser apreendida pelo processo formativo na área da saúde, para que a universidade possa cumprir sua missão institucional de relevância social.

Um marco do movimento da valorização dos trabalhadores da saúde no Brasil foi a criação da Secretaria de Gestão do Trabalho e da Educação na Saúde - SGTES, por meio do Decreto 4.726, de 9 de junho de 2003, na estrutura regimental do Ministério da Saúde (BRASIL, 2003), como uma das Secretarias finalísticas, incumbidas da formulação e implementação da política nacional de saúde. A SGTES tem como missão desenvolver políticas e programas que busquem assegurar o acesso universal e igualitário às ações e aos serviços de saúde, impondo à função da formação e da gestão do trabalho a responsabilidade pela 
qualificação dos trabalhadores e pela organização do trabalho em saúde, constituindo novos perfis profissionais com condições de responder à realidade de saúde da população e às necessidades do SUS. No plano da orientação educacional as bases para essa política seguem os princípios da política de educação e as tendências contemporâneas que tratam os processos educativos como movimento científico, técnico, ético e crítico-operacional de construção, de interação e de produção social, em que a dinâmica da aprendizagem resulta do conhecimento multidimensional e interdisciplinar e da articulação com o processo de prestação de serviços (BRASIL, 2011).

O eixo paradigmático que alinha e organiza a política de educação na saúde é a integração do ensino com a rede de prestação de serviços do SUS, instituído como ato pedagógico que aproxima profissionais da rede de serviços de saúde das práticas pedagógicas e os professores dos processos de atenção em saúde, possibilitando a inovação e a transformação dos processos de ensino e de prestação de serviços de saúde.

O Programa Nacional de Reorientação da Formação Profissional em Saúde - Pró-Saúde, implementado e executado pelo Ministério da Saúde, em parceria com o Ministério da Educação, com Instituições de Ensino Superior e secretarias estaduais e municipais de saúde, desde 2005, engloba um conjunto de iniciativas voltadas ao processo de mudança nos cursos de graduação na área da saúde (HADDAD et al., 2008).

As ações desenvolvem-se em torno de três eixos principais: o da orientação teórica, da orientação pedagógica e dos cenários de prática. Uma das estratégias que se articulam ao Pró-Saúde no eixo de cenários de prática é o Programa de Educação pelo Trabalho em Saúde - o PET Saúde (HADDAD et al., 2009), que oferece bolsas de estudo em três modalidades: tutoria (docente), monitoria (aluno de graduação) e preceptoria (profissional da Estratégia de Saúde da Família, que atua no aprendizado dos alunos de graduação, sob supervisão docente do tutor), tendo como cenário de prática a atenção primária à saúde nas
Unidades de Saúde da Família. Dessa forma, são organizados grupos tutoriais que atuam nos serviços de saúde com foco no aprendizado e na vivência dos alunos de graduação, no modelo de atenção à saúde do SUS. Com o PET Saúde, os objetivos e eixos do Pró-Saúde foram potencializados, resultando na participação mais ativa de profissionais do serviço nesse processo (preceptor bolsista) e na atuação docente orientando pedagogicamente o grupo tutorial. O PET Saúde também introduziu o componente de pesquisa, proporcionando maior reflexão sobre a prática, bem como a produção de conhecimento orientada pelos desafios da atenção e gestão nos serviços de saúde. Esse é um aspecto que confere maior relevância social à pesquisa, impacto e, muitas vezes, possibilidade da aplicação imediata dos resultados na melhoria dos serviços.

O Pró-Saúde e o PET Saúde foram implementados em todo o país, em aproximadamente mil cursos de graduação em integração com mais de mil serviços de saúde do SUS. Entre os resultados já alcançados pelos programas, verificam-se o aprofundamento da integração ensino-serviço, o aperfeiçoamento da capacidade dos estudantes de se comunicarem com seus pacientes e compreenderem a complexidade do contexto social em que se dá o processo saúde-doença, o aprendizado no processo de trabalho em equipe multiprofissional, a conquista progressiva da autonomia frente a situações clínicas de complexidade crescente, inseridas no serviço público de saúde.

É neste contexto nacional das políticas de educação e saúde que o curso de graduação em Odontologia da FOUSP conduziu uma reestruturação curricular, desde 2005, com o objetivo de atender às Diretrizes Curriculares Nacionais para os cursos de graduação em Odontologia (BRASIL, 2002). O projeto de mudanças recebeu apoio do Ministério da Saúde, em parceria com o Ministério da Educação, por meio do Programa Nacional de Reorientação da Formação Profissional em Saúde (Pró-Saúde) e do Programa de Educação pelo Trabalho em Saúde (PET Saúde), juntamente com os demais cursos da área da saúde da USP, 
numa perspectiva de construção do trabalho em saúde multiprofissional e atendendo ao princípio da integralidade da atenção à saúde no âmbito do SUS.

O currículo de graduação em Odontologia da FOUSP reestruturado retrata coerência com os objetivos das DGN e da reorientação da formação profissional proposta pelo Pró-Saúde. Entre as modificações introduzidas, o curso diurno passou de uma duração de quatro anos e meio para cinco anos, e o eixo formador clínico passou a ser transversal à matriz curricular. A clínica adquiriu a perspectiva integradora, passando a ser aberta ao SUS, em contraponto com o modelo anterior, de disciplinas estanques e isoladas umas das outras, que dificultava a construção do conceito da integralidade da atenção à saúde. Foram introduzidos os estágios vivenciais no SUS e a valorização de pesquisas orientadas para as necessidades da atenção básica, tendo a pesquisa como princípio do processo educativo, seguindo o que é também uma diretriz do Programa PET Saúde. No primeiro ano, o eixo central de formação é a Clínica Ampliada de Promoção da Saúde, e os eixos norteadores vão do geral/normal para o específico/patológico. No segundo ano, iniciam-se a Clínica Ampliada de Atenção Básica e o Núcleo Integrado de Diagnóstico. A Clínica Ampliada de Atenção Básica tem por objetivo dar continuidade às ações desenvolvidas no território, com ênfase na educação em saúde bucal, propiciando aos alunos desenvolverem junto à rede atividades educativas que ampliam o contato direto com o território.

No terceiro ano é introduzida então a Clínica de Saúde Bucal e a Saúde Coletiva. No quarto e quinto ano segue a Clínica de Saúde Bucal, agora acompanhada pela Clínica Multidisciplinar e pela Clínica de Especialidades Interdisciplinar. Os estágios vivenciais no SUS estão presentes de forma transversal ao longo de todo o curso, com graus crescentes de complexidade, estimulando o ganho gradual e progressivo de autonomia do estudante para o exercício profissional. É importante buscar avaliar o resultado efetivo e as mudanças que o novo currículo poderá propiciar com relação ao perfil dos futuros profissionais formados. A própria construção do projeto já requer da equipe de docentes envolvidos graus de abertura para a mudança, flexibilidade e desprendimento com relação às tradicionais estruturas de cargas horárias das diversas disciplinas. Fica claro também o grande desafio colocado para os docentes habituados ao ensino e à prática restritos à especialidade, de atuarem por meio de um currículo integrado, para formar um profissional generalista, crítico, capaz de atuar segundo as necessidades de saúde da população, e vinculado aos princípios do Sistema Único de Saúde (BRASIL, 2002).

A disciplina de Odontopediatria é ministrada entre o oitavo e o décimo semestre do curso de graduação, tendo tido sua duração aumentada de dois para três semestres a partir da reestruturação, embora com a manutenção do mesmo número de créditos. Trata-se de uma especialidade que tem por responsabilidade o atendimento integral, desde a promoção e a prevenção, até a reabilitação da saúde bucal do bebê, da criança e do adolescente (GUEDES-PINTO et al., 2009). Antes da reestruturação, a disciplina estava organizada com base nos conteúdos que compõem a especialidade, ministrados na forma de aulas teóricas expositivas, ao final das quais se iniciavam as atividades clínicas supervisionadas pelos docentes, envolvendo o atendimento odontológico de bebês (prevenção) e crianças, triados para o atendimento a partir do nível de complexidade compatível com a formação na graduação.

No contexto das mudanças promovidas pela política nacional de educação e de educação na saúde, que resultaram na reestruturação curricular, a nova estrutura serviu de base para que na disciplina de Odontopediatria fossem promovidas as seguintes mudanças: o planejamento pedagógico, antes embasado nos conteúdos, passou a considerar as competências e habilidades esperadas para a formação, no nível da graduação, de um cirurgião dentista generalista. O conteúdo programático foi revisto à luz dos três ciclos estabelecidos para o currículo geral do 
curso: ciclo I - diagnóstico e plano de tratamento; ciclo II - promoção e prevenção à saúde bucal e adequação do meio bucal; ciclo III - reabilitação. Considerando a importância de se promover o desenvolvimento de habilidades cognitivas, práticas (clínicas) e atitudinais, com complexidade crescente, de acordo com a taxonomia de Bloom (BLOMM, 1968), as aulas teóricas expositivas foram em parte mantidas, e complementadas com metodologias ativas no processo de ensino -aprendizagem, envolvendo a discussão de casos clínicos, a elaboração de planos de tratamento em grupo, o estudo dirigido e seminário em torno de temas selecionados. Além disso, introduziu-se a atividade laboratorial pré-clínica sobre temas para os quais a habilidade prática é imprescindível na atuação clínica. O uso das tecnologias interativas, incluindo o planejamento de atividades e a oferta de conteúdos complementares em formato multimídia na plataforma Moodle, ampliou as oportunidades de aprendizado e contato com diferentes casos clínicos. Destaca-se a mudança efetivada no processo de avaliação, antes baseado em provas teóricas e no desempenho durante as atividades clínicas. Com a reestruturação, e tendo por base o novo planejamento pedagógico, introduziu-se a prova de habilidades clínicas, por meio do denominado Objective Structured Clinical Examination (OSGE) (HARDEN, 1988; PARK et al., 2016).

Neste contexto geral da reestruturação curricular, a disciplina de Odontopediatria planejou e implementou mudanças para que seus objetivos, estratégias pedagógicas e de avaliação fossem atualizados à luz do novo contexto, tanto do curso, como da política nacional de educação na saúde. Procurou também avaliar as mudanças ao longo do seu processo de implementação.

\section{Objetivo}

Analisar comparativamente, nos dois modelos curriculares do curso de graduação em Odontologia, anterior e posterior à reestruturação, o desempenho e a percepção dos alunos de graduação sobre a disciplina de Odontopediatria e seu aprendizado.

\section{Metodologia}

Conforme mencionado, para atender às diretrizes estabelecidas no projeto de reestruturação curricular, a Disciplina de Odontopediatria da FOUSP foi reorganizada em três ciclos no novo currículo: diagnóstico e plano de tratamento; promoção e prevenção; reabilitação. Esses ciclos nortearam a reformulação e redistribuição do conteúdo programático, de tal forma que cada tópico passa a ser abordado não mais sequencialmente, mas nos três ciclos, aprofundando-se sua análise, em articulação com outros tópicos, e sob a perspectiva considerada. Como exemplo, vale citar o traumatismo dentário em Odontopediatria. No ciclo I, o tema é abordado com relação ao seu diagnóstico e conduta; no ciclo II, com relação à sua prevenção, que tem relação com as fases do desenvolvimento infantil, com a predisposição influenciada por fatores como, por exemplo, determinados tipos de maloclusão; e, no ciclo III, o tema é abordado sob a perspectiva do prognóstico e do tratamento das sequelas ou reabilitação. Foram introduzidas atividades préclínicas, com o objetivo de aproximar o conteúdo teórico da prática clínica, e o início das atividades clínicas foi antecipado e sincronizado com o conteúdo teórico e pré-clínico correspondente. Introduziu-se também o uso das tecnologias de informação e comunicação (TIC). Os alunos desenvolvem atividades aplicadas, com foco em casos clínicos, na plataforma Moodle, sendo monitorados pelos alunos de pós-graduação que recebem capacitação para atuar como tutores de educação a distância na plataforma Moodle, sob supervisão docente.

Mais especificamente, as atividades complementares desenvolvidas e aplicadas aos alunos da graduação na plataforma Moodle tiveram como referência o conteúdo programático ministrado, que foi enriquecido com casos clínicos para serem diagnosticados, artigos científicos, e vídeos demonstrativos de procedimentos clínicos. Esse material deveria ser acessado pelos alunos no horário de sua conveniência, como complemento das atividades presenciais, e é incluído 
na avaliação. A realização das atividades no prazo estabelecido e a consulta aos conteúdos são mais valorizadas na avaliação dessas atividades do que propriamente o índice de acertos.

As avaliações de ambas as turmas, a de antes e depois da reestruturação, foram feitas por meio de prova teórica e prática de habilidades clínicas calibradas (OSCE), e ao final do curso foi aplicada a técnica qualitativa do grupo focal para observar a percepção dos alunos ao final da disciplina, comparando as duas turmas (MINAYO, 2014). O grupo focal foi realizado com onze alunos voluntários do último ano da última turma do currículo antigo, e depois com outro grupo de onze alunos voluntários do último ano da primeira turma com o currículo reestruturado. Todos assinaram o Termo de Consentimento Livre e Esclarecido, e o projeto foi aprovado pelo Comitê de Ética em Pesquisa (CEP) da FOUSP (Parecer n. 1.070.697/2015).

Ambos os grupos foram conduzidos pelo mesmo moderador, docente e pesquisador com experiência na aplicação do método. $\mathrm{O}$ roteiro conteve as seguintes questões norteadoras: quais as expectativas iniciais dos participantes sobre o curso de graduação em Odontologia quando ingressaram na faculdade; se essas expectativas foram correspondidas ao final do curso; quais eram as expectativas dos participantes sobre a disciplina de Odontopediatria antes de cursá-la; se as expectativas foram correspondidas ao término da disciplina. A cada grupo foi esclarecido sobre como eram o curso e a disciplina antes e depois da reestruturação. Cada grupo tinha vivenciado uma das duas situações e procurou imaginar e compará-la com a outra (currículo e disciplina de Odontopediatria anterior e posterior à reestruturação).

As mudanças aqui relatadas foram introduzidas a partir do ano de 2012, e o estudo comparativo entre os alunos do currículo antigo e do currículo novo, aplicado no ano de 2015.

\section{Resultados}

As notas nas avaliações, variando de 1 a 10 , foram comparadas pelo teste $\mathrm{T}$, sendo que 53 alunos pertenciam ao currículo antigo e 55 ao currículo novo. As médias da prova prática foram respectivamente 6,61 e 7,08, e o desempenho global de 5,93 e 6,40 , com diferença estatisticamente significante entre os grupos $(\mathrm{p}=0,01, \mathrm{CI}=0,008-0,05$, na avaliação prática, e $\mathrm{p}=0,0017, \mathrm{CI}=0,18299$, no desempenho global). No grupo focal, a percepção dos estudantes do currículo reestruturado foi predominantemente positiva, enquanto a dos estudantes do currículo antigo foi mais crítica.

A diferença de percepção entre os estudantes do currículo anterior comparado com os do currículo reestruturado pode ser observada em alguns trechos de depoimentos.

Currículo anterior:

"O índice de desistência do curso é grande por faltar contato com a Odontologia de fato desde

\begin{tabular}{|c|c|c|c|}
\hline & \multicolumn{3}{|c|}{ Tipo de Currículo } \\
\hline & Currículo Antigo & Currículo Novo & Valor P (IC) \\
\hline \multicolumn{4}{|l|}{ Tipo de Avaliação } \\
\hline $\begin{array}{l}\text { Prova Prática Média } \\
\text { (DP) }\end{array}$ & $6,61(1,03)$ & $7,08(0,91)$ & $0,01(0,008-0,05)$ \\
\hline $\begin{array}{l}\text { Desempenho Global } \\
\text { Média (DP) }\end{array}$ & $5,93(0,67)$ & $6,40(1,01)$ & $0,0017(0,18)$ \\
\hline
\end{tabular}

Tabela 1 - Comparação dos dois tipos de currículos. DP = Desvio Padrão. Significância estatística $=p<0,05$. 
o começo do curso. Algumas matérias mais clínicas desde o início incentivariam mais os alunos a continuarem."

"A faculdade treina muito a técnica, e falta a parte sobre como se comunicar melhor com os pacientes. Ter mais conteúdo no curso sobre atitudes e questões éticas na relação com o paciente. $\mathrm{E}$ isso deveria estar presente em todas as disciplinas e não ser apenas uma disciplina específica."

"A Odontopediatria é a clínica geral voltada para a criança. Achei que seria mais focado na abordagem psicológica, e acabamos tendo só uma aula sobre isso. Eu sei o procedimento, mas não tenho segurança de sair e atender uma criança difícil, que dê trabalho."

"Quanto ao conteúdo correspondeu. Na clínica também. O tempo de clínica foi meio corrido para atender a todos os pacientes."

Currículo reestruturado:

"Gostei de ter visto desde o primeiro ano como funcionava a clínica. Como eu não tenho pais dentistas, ter visto como se faz uma prótese, um tratamento de canal, formular e esclarecer dúvidas logo no começo foi importante."

"A tendência é formar generalistas. E a reestruturação teve esse objetivo. Com relação à expectativa, o curso supriu, teve bastante coisa prática, a ligação entre clínico, teórico e prático ajuda o aprendizado."

"Na Odontopediatria aumentou o tempo de clínica, e hoje, se eu tivesse que atender uma criança, eu atenderia."

"A mudança foi em geral positiva, mas ainda há problemas. $\mathrm{O}$ mais importante foi diluir o conhecimento em mais tempo. E também retomar os conteúdos e temas ao longo do curso, isso ajuda."

\section{Discussão}

O processo de planejamento da reorganização do currículo da disciplina de Odontopediatria foi conduzido pela equipe, que se debruçou de um lado sobre o projeto de reestruturação do curso como um todo, e por outro, buscou interpretar e aplicar as DCN, ampliando o olhar para além de uma visão mais estrita dos conteúdos, e partindo para o estabelecimento de objetivos pedagógicos com base no perfil de competências esperado para o cirurgião-dentista graduado.

A ordenação dos ciclos com seus respectivos conteúdos considera o perfil profissional estabelecido pelas Diretrizes Curriculares Nacionais para os cursos de graduação em Odontologia, e as habilidades e competências a serem desenvolvidas no atendimento clínico ao paciente infantil, no seu contexto familiar e social. Leva em conta também a necessidade de planejar as atividades educacionais de forma a desenvolver nos estudantes a autonomia para a realização de procedimentos de complexidade progressiva. Foi estabelecido como objetivo geral da disciplina que "o egresso do curso seja capaz de realizar o atendimento clínico em Odontopediatria, com ênfase no diagnóstico integral, na promoção e prevenção à saúde bucal, reconhecendo e considerando as especificidades próprias do crescimento e desenvolvimento, nas suas diversas etapas: no bebê, na criança e no adolescente". O objetivo geral foi desdobrado em um conjunto de objetivos específicos. Para o alcance dos objetivos educacionais propostos, os conteúdos foram integrados na estrutura do curso, envolvendo dimensões cognitivas, afetivas e psicomotoras (taxonomia de Bloom).

As atividades complementares ao curso presencial, desenvolvidas na plataforma Moodle da rede social Stoa (http://disciplinas.stoa.usp.br/), contribuem para ampliar as oportunidades de aprendizagem explorando o potencial do uso das TIC e transcendendo os momentos presenciais em sala de aula, laboratório e clínica. Além disso, propiciam o contato dos alunos com um número maior e diversificado de casos clínicos.

Conforme pôde ser observado pelo grupo focal, o início de atividades clínicas mais precoce era uma expectativa, e que foi atendida pela reestruturação curricular. A ampliação da duração da clínica, um período de tempo mais diluído para a apreensão dos conteúdos e a ampliação das vivências durante os atendimentos também foram percebidos como mudanças positivas. 
Chama a atenção a expectativa dos estudantes de que se proporcionasse, de forma transversal no curso, mais espaço para o aprendizado sobre a comunicação com os pacientes e as questões éticas envolvidas na atenção à saúde, e na relação profissional-paciente.

A ampliação da duração da disciplina de Odontopediatria, com a diversificação e a maior dinâmica estabelecida no planejamento das atividades, foi percebida como se tivesse havido aumento do conteúdo, embora o número de créditos tenha sido mantido. Possivelmente a ampliação da duração, mas também a dinâmica das atividades, pode ter levado a um maior engajamento, motivação, e facilitado o processo de aprendizagem.

É fundamental o planejamento adequado do processo avaliativo. Nesse sentido, as provas teóricas apresentam uma abordagem problematizadora e integradora de conhecimentos, com base na aplicação clínica. O processo avaliativo realizado durante as atividades laboratoriais e clínicas foi organizado por meio de uma ficha contendo habilidades e competências que se quer desenvolver, com os seus respectivos indicadores. Essa ficha contendo os indicadores de avaliação das atividades clínicas foi adotada a partir da reestruturação, objetivando uma padronização dos critérios de avaliação do desempenho dos estudantes, bem como a coerência entre os objetivos pedagógicos e os indicadores norteadores da avaliação.

Para compor o quadro geral de notas dos alunos, são consideradas as avaliações teóricas, práticas de laboratório, clínica, a participação e o envolvimento dos alunos monitorados no ambiente virtual e-disciplinas, plataforma Moodle (Teleodontopediatria).

Um elemento inovador introduzido foi a prova prática de habilidades clínicas. São elaboradas questões envolvendo situações que os alunos enfrentarão na clínica, distribuídas por dez estações de trabalho, e em cada estação o aluno é avaliado a partir de uma lista de tarefas que descreve o desempenho esperado. Este modelo de prova é aplicado quando o aluno já percorreu boa parte das unidades de conhecimento, os laboratórios pré-clínicos, e está iniciando as atividades clínicas. Com isso, o aluno consegue identificar quais as dificuldades e os desafios a serem superados para desempenhar seu papel na clínica infantil da forma mais satisfatória possível, potencializando o alcance da vivência clínica.

A disciplina de Odontopediatria apresenta-se com algumas características que a diferenciam de várias outras disciplinas do curso. Ela se caracteriza, de certa forma, por envolver a clínica geral aplicada às diferentes etapas do crescimento e desenvolvimento infantil, mais do que propriamente uma especialidade como a endodontia e a periodontia, por exemplo. Nesse mesmo sentido, sem ser a saúde coletiva, ela representa a disciplina que mais se identifica com a atenção primária à saúde, nos seguintes aspectos: visão abrangente e integrada de promoção, prevenção e reabilitação; vínculo longitudinal entre profissional e paciente; abordagem centrada não apenas no paciente, mas considerando o núcleo familiar.

A importância deste entendimento e dessas associações reside no fato de que o processo de mudança é multidirecional e se desenvolve não apenas dentro dos limites de cada profissão, mas no campo de suas relações e com impactos nos processos de gestão da atenção e do cuidado em saúde, na compreensão ampliada do processo saúde-doença e na construção de um dos mais complexos princípios do SUS, o da integralidade da atenção à saúde.

Os depoimentos colhidos na aplicação do grupo focal retratam diferentes expectativas e percepções com relação ao curso, algo que pode ter recebido a influência da realidade específica da qual cada estudante é oriundo. A expansão e democratização do acesso à educação superior promovida pelo conjunto de iniciativas formado pelo Prouni, Fies, Reuni e cotas permitiram a chegada à universidade de estudantes provenientes de contextos socioeconômicos diversos, de modo especial em cursos historicamente mais elitizados como é o caso de Odontologia e Medicina, por exemplo. Recebê-los e acolhê-los em condições de equidade mostra-se ainda um desafio colocado para a comunidade acadêmica da educação superior brasileira. 


\section{Conclusões}

A reorientação da formação na graduação em saúde deve atender, de forma abrangente, à política nacional de educação e à política nacional de saúde, considerados a relevância social e o papel da universidade no desenvolvimento social e econômico do país. O curso de graduação em Odontologia da FOUSP implementou, a partir de 2005, as mudanças necessárias conforme projeto submetido e aprovado em editais do Pró-Saúde e PET Saúde, e também preconizado pelas DCN. A disciplina de Odontopediatria reorganizou seu currículo para melhor atender à reestruturação curricular implementada, e avaliou o desempenho e a percepção dos estudantes em relação às mudanças implementadas. Os estudantes do currículo reestruturado apresentaram melhor desempenho nas avaliações e tiveram uma percepção mais positiva sobre o curso da disciplina de Odontopediatria, comparados com os estudantes do currículo anterior à reestruturação.

\section{Agradecimentos}

Agradecemos aos docentes da disciplina de Odontopediatria da FOUSP, Profs. Drs. Maria Salete Nahás Pires Correa, Fausto Medeiros Mendes, Mariana Minatel Braga, Márcia Wanderley e José Carlos Imparato, pelo trabalho de equipe desenvolvido, a partir do qual este estudo foi realizado.

\section{Referências Bibliográficas}

BARDIN, L. Análise de Conteúdo. Lisboa: Edições 70, 2011.

BLOOM, B. Learning for Mastery. Los Angeles: Center for the Study of Evaluation of Instructional Programs UCLA, 1968.

BRASIL. Ministério da Educação. Conselho Nacional de Educação/Câmara de Educação Superior. Resolução N. 3 de 19 de Fevereiro de 2002. Disponível em: <http://portal.mec.gov.br/cne/arquivos/pdf/ CES032002.pd† $>$. Acessado em 8 abr. 2018.
BRASIL. Ministério da Educação. Lei Federal N. 10.861 de 14 de Abril de 2004 (Sistema Nacional de Avaliação da Educação Superior - Sinaes). Disponível em: <http://www.planalto.gov.br/ccivil_03/_ato20042006/2004/lei/l10.861.htm>. Acessado em 8 abr. 2018.

BRASIL. Ministério da Educação. Lei N. 13.005 de 25 de Junho de 2014 (Plano Nacional de Educação 2014-2024 - PNE). Disponível em: <http://www.planalto.gov.br/ccivil_03/_ato2011-2014/2014/lei/113005. htm>. Acessado em 8 abr. 2018.

BRASIL. Ministério da Saúde. Decreto N. 4.726 de 9 de Junho de 2003 (Aprova a Estrutura Regimental e o Quadro de Cargos em Comissão e Funções Gratificadas e Dá Outras Providências). Disponível em: $<$ http://www.planalto.gov.br/ccivil_03/decreto/2003/ D4726.htm >. Acessado em 8 abr. 2018.

BRASIL. Ministério da Saúde. Secretaria de Gestão do Trabalho e da Educação na Saúde. Políticas e Ações. Brasília, 2011. Disponível em: <http://docplayer.com. br/1248040-Ministerio-da-saude-secretaria-de-gestaodo-trabalho-e-da-educacao-na-saude-sgtes-politicas-e-acoes.html >. Acessado em 2 abr. 2016.

GUEDES-PINTO, A. C.; BONEGKER, M. \& RODRIGUES, C. R. D. (orgs.). Fundamentos de Odontologia: Odontopediatria. São Paulo: Santos Editora, vol. 1., 2009.

HADDAD, A. E. A Odontologia na Politica Nacional de Formação dos Profissionais de Saúde, o Papel da Teleodontologia como Ferramenta no Processo de Ensino-Aprendizagem e a Criação do Núcleo de Teleodontologia da FOUSP. Tese de Livre-Docência em Odontopediatria. Faculdade de Odontologia, Universidade de São Paulo, 2011. 202p. Disponível em: <http://www.teses.usp.br/teses/disponiveis/livredocencia/23/tde-13052013-143506/pt-br. php>. Acessado em 8 abr. 2018.

; BRENELLI, S.; PASSARELLA, T. M. \& CAMPOS, T. "Política Nacional de Educação na Saúde". Rev Baiana Saúde Pública, 32, supl. 1, 2008, pp. 98-114.

$\mathrm{E}$ "Progra,$\longrightarrow$ $\longrightarrow$ PET Saúde". Cadernos Assoc Bras Educacãa Médica, n. 5, 2009, pp. 6-12.

HARDEN, R. M. "What is an OSCE?". Med Teacher, vol. 10, n. 1, pp. 19-22, 1988.

MINAYO, M. C. S. O Desafio do Conhecimento: Pesquisa Qualitativa em Saúde. São Paulo: Haucitec, 2014.

PARK, S. E.; ANDERSON, N. K.; \& KARIMBUX, N. Y. "OSCE and Case Presentation as Active Assessment of Dental Student Performance".J Dent Educ, n. 30, 2016, pp. 334-38. 